\title{
A New Rotary Hexapod for Micropositioning
}

\author{
Jonathan Coulombe and Ilian A. Bonev \\ École de technologie supérieure \\ Montreal, Quebec, Canada \\ ilian.bonev@etsmtl.ca
}

\begin{abstract}
Hexapods are widely used for precision positioning, but their workspace is too limited for some applications. Often, a rotary stage is added to enlarge the orientation workspace, but this increases the system cost. As an alternative, rotary hexapods have been proposed in which the legs are of fixed length and the base attachment points slide along a common circular path. However, these hexapods have a reduced range of motion in some degrees of freedom due to mechanical interferences. In contrast, we propose a design in which two concentric circular rails are used in addition to double spherical joints, three extra rotary sensors, and two highaccuracy rotary encoders. As a result, the workspace of our robot is significantly increased, its direct kinematics can be solved linearly, and its high accuracy is guaranteed. A prototype has been built and is described in detail.
\end{abstract}

Keywords-hexapod; parallel robot; precision robotics;

\section{INTRODUCTION}

Most robotic systems for six-axis precise positioning are based on hexapods. Today, the key manufacturers of precision hexapods are PI and PI MiCos (both in Germany) and Symétrie (in France). Newport and ALIO Industries (both in USA) also manufacture precision hexapods. Nearly all commercial precision hexapods have telescoping legs.

It is well known that hexapods with telescoping legs have small workspace. Typically, these hexapods offer no more than $\pm 30^{\circ}$ rotational capabilities. In some applications, this is clearly not enough, and both PI and ALIO Industries have delivered custom-made rotary hexapods (with telescoping legs) that are either mounted on a rotary stage or in which a rotary stage is attached to the mobile platform.

An ingenious alternative to hexapods combined with a rotary stage was patented by a team of Russian scientists in 1983 [1]. This hexapod, discussed in detail in [2], consists of six legs of fixed length that are attached to six carriages sliding independently along a common circular rail. The legs are attached to the mobile platform in pairs, each pair connected through a revolute joint, and then through a ball joint, as shown in Fig. 1a. A very similar design, the Rotopod, was also patented in 1999 [3] by Hexel Corporation, currently Mikrolar (in the USA). The main difference is that the legs of the Rotopod are attached to the mobile platform through double spherical joints, as shown in Fig. 1b. The first Rotopod prototypes actually followed the design of [1], but the company switched promptly to a simpler mechanical design with twelve ball joints (Fig. 2) manufactured by Hephaist Seiko (in Japan).
Since the Rotopod uses a single circular rail, adjacent carriages interfere as soon as the mobile platform is lowered approximately $50 \mathrm{~mm}$, for their smaller R 1000 model, which has a footprint of $1 \mathrm{~m}$ in diameter. As a comparison, Symétrie's SIRIUS telescoping-legs hexapod is smaller, yet has a position workspace that is nearly twice that of R 1000 . Furthermore, the twisting of the six motor cables prevents the Rotopod from rotating endlessly about a vertical axis.

An alternative design providing endless rotary motion was patented in 2011 [4] and is currently commercialized by CEMEC (in Germany) under the name Rotapod (Fig. 3). However, while the Rotapod has large orientation workspace, its position workspace is still relatively small.

In order to improve the workspace of the rotary hexapod, we developed a design with two concentric circular rails and double spherical joints (Fig. 4). Furthermore, unlike other rotary hexapods, we use high-accuracy optical rotary encoders as well as three extra rotary sensors, which makes it possible to compute the direct kinematics linearly.

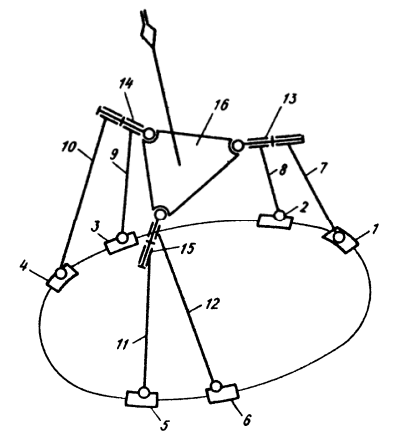

(a)

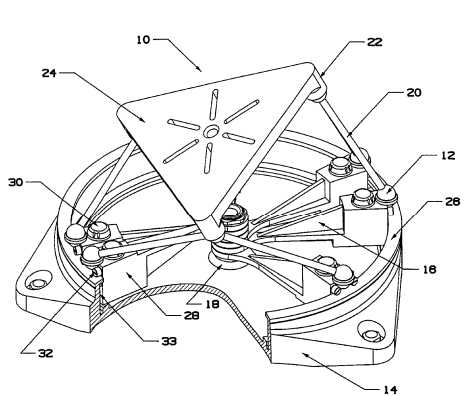

(b)
Figure 1. The first two rotary hexapods (sources [1] and [2])

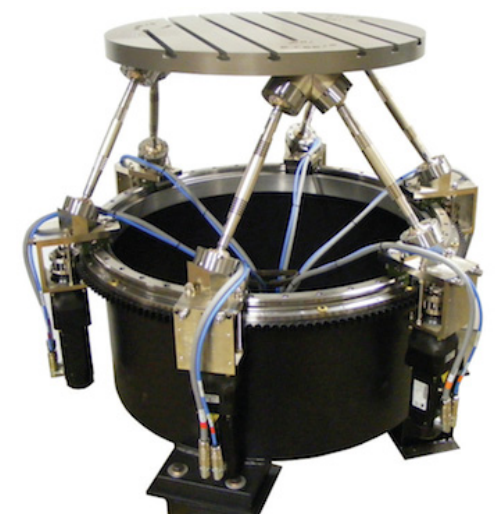

Figure 2. Mikrolar's R Series Rotopod (courtesy of Mikrolar) 

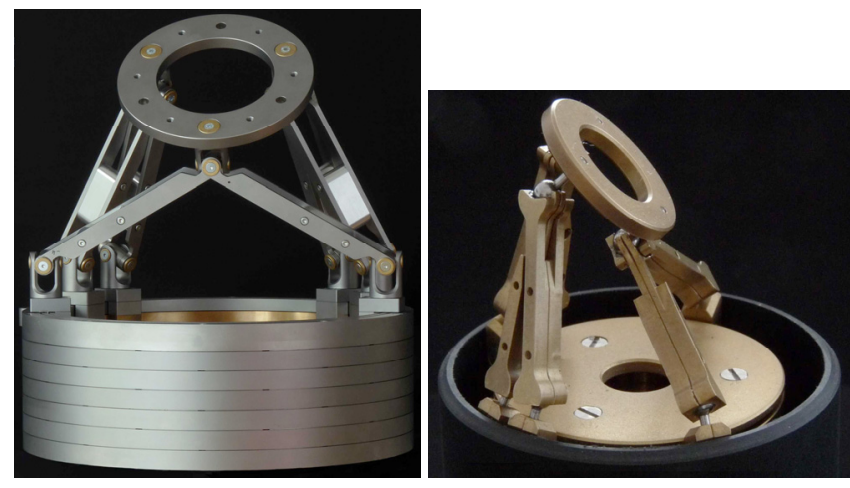

Figure 3. CEMEC's two Rotapod models (courtesy of CEMEC)

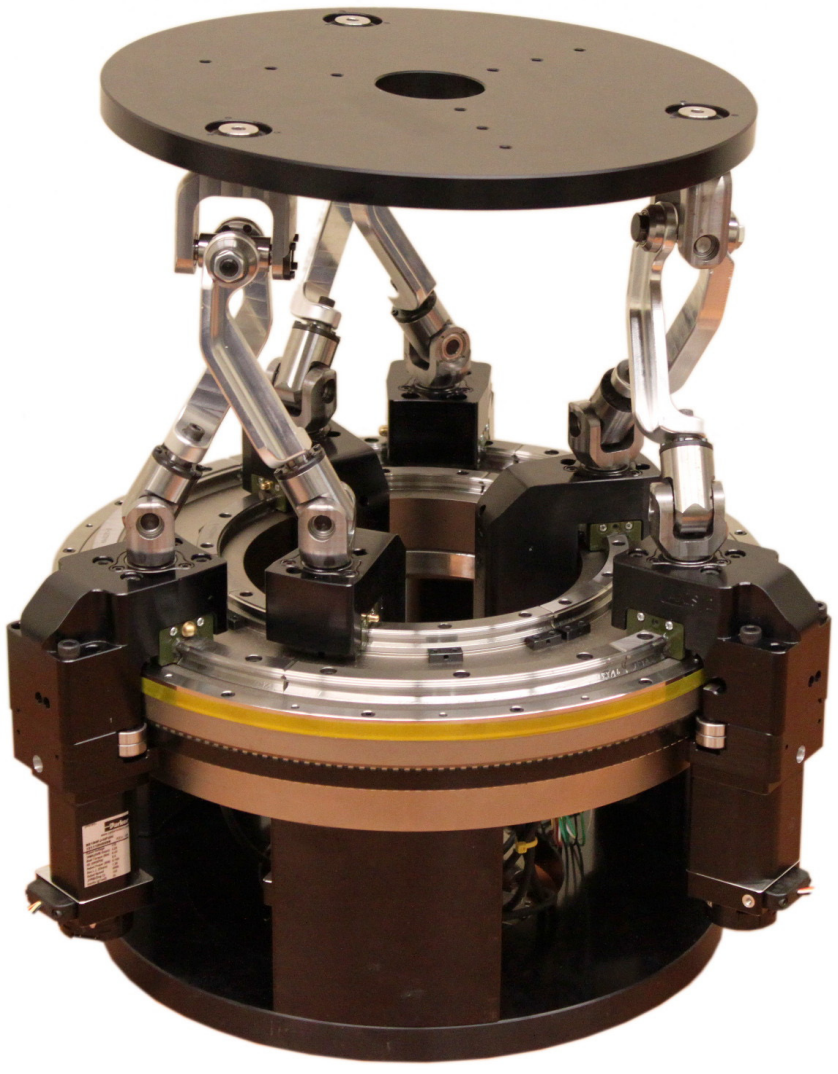

Figure 4. Photo of our MicARH (see also http://youtu.be/B1YEAfjTbus)

Section II presents the mechanical design of our rotary hexapod. Its kinematics is then described in Section III, while its workspace is described in Section IV. Conclusions and plans for future works are given in Section V.

\section{Mechanical Design of MicARH}

The first prototype of our precision rotary hexapod named MicARH (Micropositioning Agile Rotary Hexapod) is shown in Fig. 4. The choice of rotary guides was the most crucial yet the simplest to make. Indeed, there seems to be only one product available: the HCR series manufactured by THK. Mikrolar's smaller Rotopod models use the same rotary guides. Since we wanted to develop an alternative to small hexapods such as PI's M-850 and Newport's HXP100, we chose the two smallest guides from the THK HCR line.,
We purchased one HCR12A3UUC1+60/100RH-6T circular guide and one HCR15A3RRC1+60/150RH-6T, which have a radius of $100 \mathrm{~mm}$ and $150 \mathrm{~mm}$, respectively. These rails come in $60^{\circ}$ segments fitted at the factory to obtain a full circle. The LM guide of each carriage is preloaded to eliminate any backlash.

The inner and outer carriage blocks, which support the motor, leg base joint and encoder sensor, are mounted on each corresponding LM guide. The actuation is accomplished through two synchronous timing pulleys fixed to the base of the robot (one for the inner rail and one for the outer rail) and a driving pulley is mounted onto the servo motor of each carriage. Despite the fact that a belt element can reduce the overall stiffness of the robot, it has the advantage of yielding a compact and backlash-free drive system. The belt play is adjusted by two rollers in each block. The inner carriages use an MXL size belt and their final drive ratio is 9:157. The outer carriages block mounted on the outside are driven by an XL size belt with a final drive ratio of 1:21.

It is interesting to note that Mikrolar's latest Rotopod model, the R 3000, uses Nexen's Roller Pinion Gear (RPG) which is a roller gear drive with less than \pm 7 arcsecs of backlash (http://shar.es/ulx5o). Naturally, we considered that option but discarded it promptly because Nexen does not offer small RPGs suitable for our dimensions.

As for the motors, we chose the smallest, $41 \mathrm{~mm}$, servo motor from Parker's BE series. More precisely, we retained the BE164FJ model for its dynamic performance.

Our choice of position feedback device is what sets us apart from other rotary hexapods. We use Renishaw's RESM encoder rings, RESM20USA209 and RESM20USA350, paired with six TONiC optical readheads. The encoder rings are directly mounted on the robot's base (the outer one visible in Fig. 4, but covered with a protective yellow tape). Thus, the accuracy of our hexapod does not rely on motor encoders or on the drive system. The ring encoders are conventional optical encoders with a graduation accuracy of $\pm 0.5 \mu \mathrm{m}$. The output from the encoder readhead is made of two sinusoidal signals with phase quadrature. We developed our own interface that interpolates the signal from the readheads, with the use of high-performance analog-todigital converters. With the actual graduation pitch of the encoder pattern, which is $20 \mu \mathrm{m}$, we were able to get a resolution of $0.315 \mu \mathrm{m}$.

Each leg is connected to its carriage via a spherical joint developed in-house based on the conventional gimbal design. The legs are also connected in pairs to the mobile platform through a scissors-like spherical joint, also developed in-house. All joints are machined from highstrength alloy steel. The joints and the shape of the legs were designed to avoid mechanical interferences as much as possible.

Another advantage of our joint design is that we were able to put an absolute encoder on the vertical pivot of each inner carriage. These low-cost encoders use a Hall-effect sensor to detect the orientation of a magnet fixed on the joint pivot and will be used to solve linearly the direct kinematic problem. 
Finally, the geometric parameters of MicARH are as follows. The length of all legs (measured between the spherical joint centers of each leg) is $129.320 \mathrm{~mm}$. The radius of the circle passing through the centers of the inner base joints is $100.010 \mathrm{~mm}$ and the radius of the circle passing through the centers of the outer base joints is $150.810 \mathrm{~mm}$. The radius passing though the centers of the platform joints is $125.410 \mathrm{~mm}$. The length of the legs and the size of the mobile platform were obtained by a preliminary optimization seeking to maximize the workspace of the hexapod The final design will be determined after a series of tests on the current prototype and using interval analyses.

\section{INVERSE AND DIRECT KINEMATICS OF MICARH}

\section{A. Inverse Kinematics and Singularities}

The inverse kinematics for the leg of any Rotopod was presented in detail in [5]. We will therefore present only the results. Let $\theta_{i}(i=1,2, \ldots, 6)$ be the active-joint variables, as shown in Fig. 5. We have two solutions defined as

$$
\theta_{i}=\operatorname{atan} 2\left(\frac{p_{i} y_{B_{j, j+1}}+x_{B_{j, j+1}} \delta_{i} \sqrt{\Gamma_{i}}}{\sqrt{x_{B_{j, j+1}^{2}}^{2}+y_{B_{j, j+1}}^{2}}}, \frac{p_{i} x_{B_{j, j+1}}-y_{B_{j, j+1}} \delta_{i} \sqrt{\Gamma_{i}}}{\sqrt{x_{B_{j, j+1}}^{2}+y_{B_{j, j+1}}^{2}}}\right),
$$

where $j=i$, if $i$ is odd, else $j=i-1$, and $x_{B_{j, j+1}}$ and $y_{B_{j, j+1}}$ are the $x$ and $y$ the coordinates of the center of the platform spherical joint $B_{j, j+1}$, with respect to the base reference frame $O-x y z$,

$$
\begin{gathered}
p_{i}=\frac{\left\|\mathbf{r}_{O B_{j, j+1}}\right\|^{2}+\left\|\mathbf{r}_{O A_{i}}\right\|^{2}-\ell^{2}}{2\left\|\mathbf{r}_{O A_{i}}\right\|}, \\
\Gamma_{i}=x_{B_{i}}^{2}+y_{B_{i}}^{2}-p_{i}^{2},
\end{gathered}
$$

$\ell$ is the length of the legs and $\delta_{i}= \pm 1$ is the branch index.

When the center of a platform spherical joint lies on the $z$ axis, (i.e., the axis of symmetry of both circular rails), (1) is not defined and the carriages of the legs connected to that joint can slide endlessly even if the mobile platform is stationary. This situation, however, cannot occur in the actual prototype of MicARH because all legs have equal length.

Another serial singularity can occur when a leg is vertical. In that case, the base spherical joint is in a singularity (known as gimbal lock) and can rotate freely. However, this situation cannot occur either because all legs have equal length.

Therefore, of all serial singularities, MicARH can only have the common type, where a leg lies in a plane passing through the $z$ axis, but is neither vertical not the center of its platform joint lies on the $\mathrm{z}$ axis. We intend to cross these singularities in order to further increase the workspace of MicARH, the way we did with our five-bar parallel robot [6] (http://youtu.be/dnixuCu49o4).

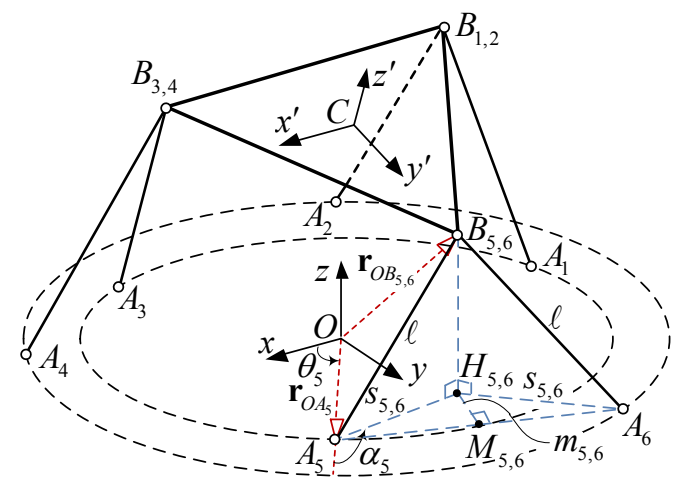

Figure 5. Schematic of MicARH

As for parallel singularities, it is no longer possible to find them analytically as we did in [5] in the case of a single circular rail. These are therefore found numerically.

\section{B. Direct Kinematics}

The direct kinematics of Mikrolar's Rotopod (Fig. 2) cannot be solved analytically. In contrast, the direct kinematics can be solved in closed-form in the case of CEMEC's Rotapod and in the case of our MicARH. However, we will still need to solve a univariate polynomial of degree eight and we will not have any automatic way of knowing the current assembly mode. For that purpose, we added an absolute rotary encoder to measure the angle of the first revolute joint of each of the three inner legs (the joints attached to the carriages of the inner circular rail).

Let $H_{j, j+1}$ be the projection of $B_{j, j+1}$ onto the $x y$ plane. Since all legs are of equal length, the distance between $H_{j, j+1}$ and both $A_{j}$ and $A_{j+1}$ is the same and will be denoted by $s_{j, j+1}$. Let also $M_{j, j+1}$ be the center of the line segment $A_{j} A_{j+1}$, and $m_{j, j+1}$ be the distance from $M_{j, j+1}$ to $H_{j, j+1}$. Finally, let $\alpha_{j}$ be the angle between vector $O A_{j}$ (denoted as $\mathbf{a}_{j}$ ) and vector $A_{j} H_{j, j+1}$. Therefore, we can find $s_{j, j+1}$ from the following system of linear equations:

$$
\begin{aligned}
\mathbf{r}_{A_{j} H_{j, j+1}} & =\frac{s_{5,6}}{\left\|\mathbf{a}_{j}\right\|}\left[\begin{array}{ccc}
\cos \alpha_{j} & -\sin \alpha_{j} & 0 \\
\sin \alpha_{j} & \cos \alpha_{j} & 0 \\
0 & 0 & 1
\end{array}\right] \mathbf{a}_{j}=\mathbf{r}_{A_{j} M_{j, j+1}}+\mathbf{r}_{M_{j, j+1} H_{j, j+1}} \\
& =\frac{1}{2}\left(\mathbf{a}_{j+1}-\mathbf{a}_{j}\right)+\frac{m_{5,6}}{\left\|\mathbf{a}_{j+1}-\mathbf{a}_{j}\right\|}\left[\begin{array}{ccc}
0 & -1 & 0 \\
1 & 0 & 0 \\
0 & 0 & 1
\end{array}\right]\left(\mathbf{a}_{j+1}-\mathbf{a}_{j}\right)
\end{aligned}
$$

where $m_{j, j+1}$ can be positive or negative. Once we know $s_{j, j+1}$, we can calculate the coordinates of point $B_{j, j+1}$ as

$$
\mathbf{r}_{O B_{j, j+1}}=\mathbf{r}_{O A_{j}}+\mathbf{r}_{A_{j} H_{j, j+1}}+\sqrt{\ell^{2}-s_{5,6}^{2}}\left[\begin{array}{l}
0 \\
0 \\
1
\end{array}\right] .
$$

Given the coordinates of all three platform joint centers, we can calculate the position and orientation of the mobile platform. 
Since our extra sensors are not very accurate, we will use this solution only as an initial estimate for a NewtonRaphson iterative procedure.

\section{POSITION WORKSPACE OF MicARH}

In [5], we presented a geometric procedure implemented in Matlab for computing the position workspace (also called constant-orientation workspace) of the Rotopod of Fig. $1 \mathrm{~b}$. We also showed that the singularity loci (i.e., the surfaces corresponding to parallel singularities) in the position workspace can be described analytically as quartic surfaces. Unfortunately, the singularity surfaces of our rotary hexapod cannot be obtained analytically. Furthermore, most of the boundaries of the position workspace of our hexapod are defined by mechanical interferences that cannot be modeled using simple geometric entities. Therefore, we use a standard ray-propagation discretization method in order to compute both the boundaries of the position workspace and the singularities surfaces inside that workspace.

We test only the interferences between carriages. The spherical joints do not limit the hexapod's workspace and leg interferences occur only at high tilt angles. As for the parallel singularity loci, we test the sign of the determinant of the Jacobian matrix (which was presented in [5]).

The position workspace of MicARH is shown in Fig. 6, for the zero orientation, and in Fig. 8, for a tilt angle of $15^{\circ}$. The green areas of the workspace boundary correspond to serial singularities, the yellow portions to carriage-tocarriage interference, and the red zones to parallel singularities.

Preliminary analyses show that the workspace of MicARH is nearly the same as that of Mikrolar's smallest Rotopod, which is at least twice as big.

\section{CONCLUSIONS AND Future WORKS}

We demonstrated that it is possible to design a rotary hexapod that has a larger workspace and potentially higher accuracy compared to similar existing prototypes. While our design uses two circular rails, these are much smaller than the rails of a single-rail rotary hexapod having a similar position workspace, and hence not necessary more expensive. Through the use of three low-cost extra rotary sensors, we can also solve the direct kinematics of our hexapod in linear form, which is not possible in other rotary hexapods.

The next step will be to perform a preliminary calibration of the hexapod using a CMM in order to evaluate the mechanical design of the robot and make all necessary adjustments. Then, we will start developing the hexapod controller. Once, the controller is completed, we will evaluate the actual workspace of the robot and recalibrate the robot. Finally, we will define an optimization criterion and optimize the size of the mobile platform and the lengths of the legs.

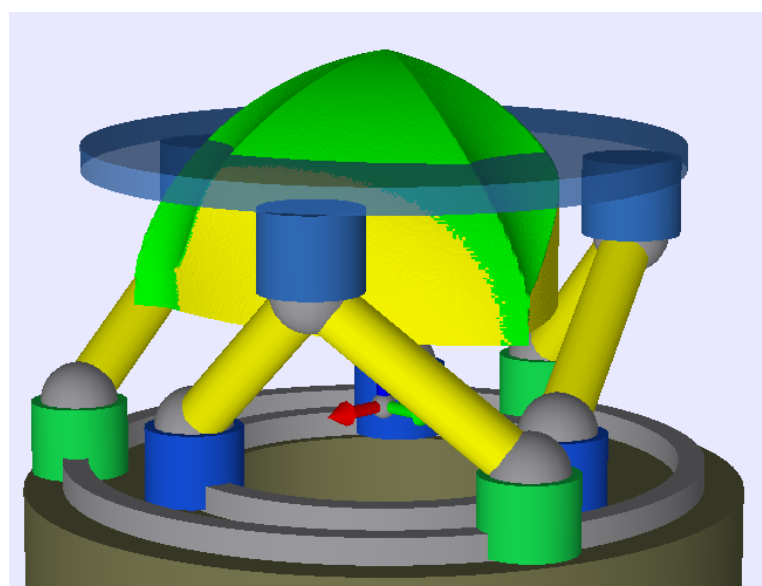

Figure 6. Position workspace of MicARH for the zero orientation

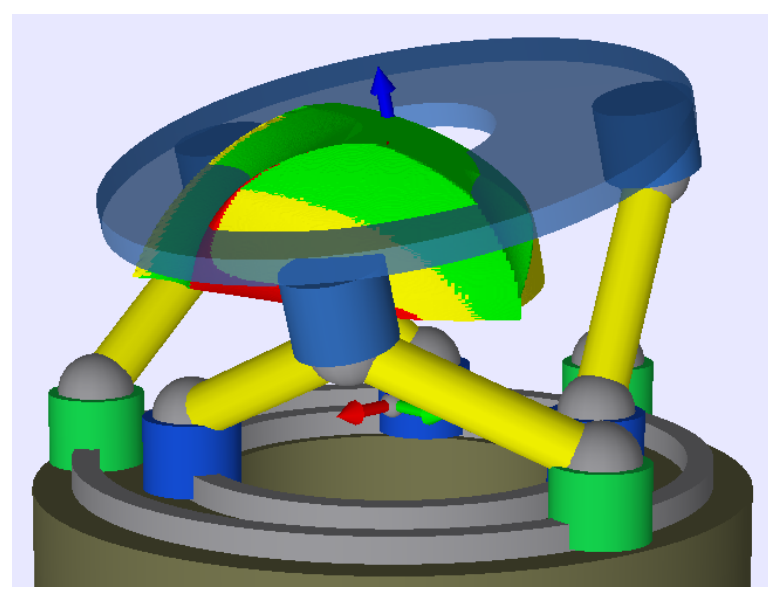

Figure 7. Position workspace of MicARH for a tilt angle of $15^{\circ}$

\section{REFERENCES}

[1] V. T. Belikov, N. A. Vlasov, K. I. Zablonski, A. M. Koritin and B. M. Shchokin, Manipulator, USSR Patent No. SU 1049244 A, 1983.

[2] B. Shchokin and F. Janabi-Sharifi, "Design and kinematic analysis of a rotary positioner," Robotica, Vol. 25, No. 1, 2007, pp. 75-85.

[3] Y. L. Chi, Systems and Methods Employing a Rotary Track for Machining and Manufacturing, US Patent No. 6,196,081, 2001.

[4] M. Schwab, Hexapod, WIPO Patent No. WO2011089198, 2011.

[5] I. A. Bonev and C. M. Gosselin, Geometric algorithms for the computation of the constant-orientation workspace and singularity surfaces of a special 6-RUS parallel manipulator," Proceedings of the ASME 2002 Design Engineering Technical Conferences, Montreal, QC, Canada, 2002.

[6] L. Campos, F. Bourbonnais, I. A. Bonev, and P. Bigras, "Development of a five-bar parallel robot with large workspace," Proceedings of the ASME 2010 International Design Engineering Technical Conferences, Montreal, QC, Canada, 2010.

[7] C. Innocenti and V. Parenti-Castelli, "Direct position analysis of the Stewart platform mechanism," Mechanism and Machine Theory, vol. 25, no. 6, 1990, pp. 611-621. 